\title{
Associations between physical and psychosocial factors and health-related quality of life in women who gave birth after a kidney transplant
}

This article was published in the following Dove Press journal: International Journal of Women's Health

\author{
Yuki Yoshikawa ${ }^{1,2}$ \\ Junji Uchida ${ }^{3}$ \\ Chiharu Akazawa ${ }^{4}$ \\ Nobuhiko Suganuma ${ }^{2}$ \\ 'Faculty of Medicine, Osaka City \\ University, Osaka, Japan; ${ }^{2}$ Department \\ of Human Health Sciences, Kyoto \\ University Graduate School of \\ Medicine, Kyoto, Japan; ${ }^{3}$ Department \\ of Urology, Osaka City University \\ Graduate School of Medicine, Osaka, \\ Japan; ${ }^{4}$ Faculty of Nursing, Osaka \\ Medical College, Osaka, Japan
}

Purpose: Health-related quality of life (HRQOL) among kidney transplant recipients is associated with physical and psychosocial characteristics. Furthermore, pregnancy and childcare may be particularly challenging for women. The aim of this study was to assess the relationship between patients' psychosocial characteristics and HRQOL, specifically for recipients who have given birth after their kidney transplant.

Patients and methods: This was a cross-sectional study. Participants were 59 kidney transplant recipients who had given birth after transplantation. The tools used were the Medical Outcomes Scale, the Kidney Transplantation Self-Management Scale, the Multidimensional Scale of Perceived Social Support (MSPSS), and The Maternal Consciousness Scale.

Results: Mean age was $42.3 \pm 7.2$ years, and the mean age at the time of transplant was $28.2 \pm 4.6$ years. A total of 82 fetal outcomes were evaluated. Maternal age was 33.6 \pm 4.1 years, duration of gestational period was $35.3 \pm 3.3$ weeks, and birth weight was $2,303.8 \pm 592.5 \mathrm{~g}$. HRQOL results were nearly the same as stratified national norms. The physical component summary was positively correlated with the MSPSS $(p=0.025)$, and self-care behavior was positively correlated with the mental component score $(p=0.029)$ and MSPSS $(p=0.016)$. A structural equation model revealed that self-care behavior and the patient-health professions partnership indirectly affected physical health through social support.

Conclusion: Self-management indirectly affects physical health through social support. To create a supportive environment through monitoring and consultation with patient families, childrearing kidney transplant recipients should be encouraged to improve their self-management skills to improve their quality of life. Social support for self-management may contribute to improve HRQOL for women who experience pregnancy and child-rearing after transplantation.

Keywords: kidney transplant, quality of life, pregnancy, child-rearing, social support

\section{Introduction}

Kidney transplant recipients require continuous drug treatment and self-management for the rest of their lives. For mothers who experienced transplants, pregnancy and childcare can have significant consequences on quality of life (QOL). Health-related QOL (HRQOL) among kidney transplant recipients is associated with several physical and psychosocial characteristics. ${ }^{1-6}$ The National Transplantation Pregnancy Registry provides data on the number of pregnancies and deliveries after organ transplantation in the USA. ${ }^{7}$ Pregnancy after kidney transplantation, as compared to other organ transplantations, is the most frequent ${ }^{7}$ because kidney transplants are the most common form of transplants worldwide. Given that the effects of pregnancy on cardiovascular functioning are quite robust, pregnancy after a kidney transplant can be quite risky. ${ }^{8-11}$
Correspondence: Yuki Yoshikawa

Skills Simulation Center, Faculty of Medicine, Osaka City University, I-4-3 Asahimachi, Abeno-ku, Osaka 545-8585, Japan

$\mathrm{Tel}+8 \mathrm{I} 66645348 \mid$

Fax +81666461985

Email zippyl03yukky@gmail.com 
Currently, the number of kidney transplant cases is 1,600 per year in Japan, and this number is consistently increasing. ${ }^{12}$ Kidney transplantation is the preferred treatment for end-stage renal disease, as it is associated with better QOL and greater longevity when compared with dialysis. ${ }^{6}$ One of the benefits of kidney transplantation is restoration of pituitary-ovarian function and fertility among women of childbearing age. ${ }^{13}$ Typically, fertility is restored within a few months to 1 year post-operation. ${ }^{8,10}$ Several kidney transplant recipients are counseled to wait for 2 years between transplantation and pregnancy, ${ }^{9-11}$ as transplantation increases the risk for obstetric complications, including hypertension, gestational diabetes, preeclampsia, maternal infections. ${ }^{7-11}$ For transplant recipients, more pregnancies end in preterm delivery or cesarean sections as compared to non-transplant healthy women..$^{7-11}$ Furthermore, kidney transplantation increases the risk for allograft loss. Since graft failure is a substantial problem after kidney transplantation, ${ }^{7,8}$ it is necessary for the recipients to be monitored and counseled both before and during pregnancy.

In addition to physical health logistics, maintaining QOL is important for these women after childbirth. QOL is the combination of physical, psychological, and social health domains. ${ }^{14}$ Several authors have investigated the effects of HRQOL following kidney transplantation. ${ }^{1-6}$ Factors affecting HRQOL following kidney transplantation include marital status, ${ }^{3}$ self-respect, ${ }^{15}$ employment,,${ }^{4,5}$ and positive social support. ${ }^{15}$ The physical domain of HRQOL is different for men and women. For instance, women report a greater decrease in physical HRQOL than men, and they also report lower scores on several physical domains with advanced age. ${ }^{2}$ Overall, HRQOL among long-term kidney transplant recipients is poorer than what is observed in the general population. ${ }^{4}$ Due to lower levels of serum creatinine, both physical and mental HRQOL are negatively impacted by kidney transplants. ${ }^{6}$ As time passes, self-care behaviors (eg, kidney protection, self-monitoring, and stress management) diminish among transplant recipients. Typically, this is because recipients lose interest in these behaviors. ${ }^{16}$ The Kidney Transplantation Self-Management Scale is traditionally employed to measure self-management behaviors. ${ }^{1}$ This scale has three dimensions, including self-care behavior, patient-provider partnership, and problem-solving. ${ }^{1}$ Overall, efficacious selfcare behaviors are directly and positively related to positive outcomes for kidney transplant recipients. ${ }^{1}$

Views on motherhood are unique in Japan. Traditionally, proof of motherly love is exemplified through a mother engaging in self-sacrificial and familial dedication behaviors. ${ }^{17}$
To this end, mothers are expected to take the prominent role in child-rearing, with very little participation from fathers. ${ }^{18}$ Therefore, maternal role recognition may be a significant component for HRQOL for several women.

While several mothers engage in responsible pregnancies (ie, taking care of their physical condition to have a healthy pregnancy), others (including kidney transplant recipients) with demonstrably poor self-care behaviors require social support for managing their child-rearing responsibilities. Taken together, we can assume that the levels of current HRQOL are greatly influenced by whether a recipient gives birth after a kidney transplant. However, no prior reports have examined HRQOL among recipients who have delivered a child since their transplant. Thus, we hypothesized that social support and maternal role recognition would be positively and significantly related with self-management, and that self-management would improve QOL for women who had given birth since their transplant.

\section{Patients and methods}

\section{Study design and participants}

We performed a prospective, cross-sectional study with adult female kidney transplant recipients. The study was conducted from July 2015 to April 2016. Prior data were not available on the number of recipients who had given birth after a kidney transplant in Japan. Accordingly, we obtained data from kidney transplant centers in Japan. We initially assumed that the number of postoperative births would be rather small. Therefore, our initial statistical models were derived based on a low number of observations. Patients who had given birth since their kidney transplant were recruited from 200 kidney transplant centers. We obtained cooperation from 21 transplant centers that had eligible patients. We were able to recruit 83 participants. When visiting an outpatient center, the medical staff explained the study and distributed questionnaires. Recipients returned their responses via mail. The participants provided written informed consent while submitting their response to questionnaires. Participants were included if they met the following criteria: transplant took place in Japan, pregnancy and delivery occurred after transplantation, and patients had no medical record of any other physical or psychiatric disorder.

Two hypotheses were addressed: 1) social support and maternal role recognition would be positively and significantly related to self-management and 2) self-management would be positively and significantly related to QOL.

The study was approved by the Kyoto University Graduate School and Faculty of Medicine Ethics Committee and, 
therefore, has been performed in accordance with the ethical standards laid down in an appropriate version of the 2000 Declaration of Helsinki as well as the Declaration of Istanbul 2008.

\section{Data collection}

\section{Demographic data}

Demographic data included age, date of kidney transplantation, date of delivery, serum creatinine level, education, employment status, donor type, number of children, and number of family members. Furthermore, data in relation to fetal outcomes, such as maternal age, duration of gestational period, and birth weight, were collected.

\section{Health-related quality of life}

HRQOL was measured with the Medical Outcomes Scale, short form (SF-36). ${ }^{14}$ HRQOL has long been employed in kidney transplant studies. ${ }^{1-6}$ The SF-36 measures health characteristics and consists of eight concept scales: physical functioning, role physical, bodily pain, general health perceptions, vitality, social functioning, role emotional, and mental health. Furthermore, the eight scales can be summarized into three components: the physical component summary (PCS), the mental component score (MCS), and the role/ social component score (RCS). These eight concept scales and three component summary scores have been normed to create a population-based score of $50 .{ }^{14}$ Internal consistency for each concept scale is $>0.70 .{ }^{14}$

\section{The Kidney Transplantation Self-Management Scale}

The Kidney Transplantation Self-Management Scale is used to measure self-management. ${ }^{1}$ The scale is composed of 27 items and is divided into three dimensions: problemsolving, self-care behavior, and patient-provider partnership. All subscales are evaluated on a 5-point Likert scale $(0-4)$. High scores indicate more adaptive behavior, and the dimensions are determined by the total score combined across each item. A factor analysis was conducted to verify the factor structure of self-management behaviors for kidney transplant recipients. Since the Kidney Transplantation Self-Management Scale was translated into Japanese, it was necessary to confirm its reliability. Regarding the factor structure of self-management behavior of the recipients, the item "When blood sugar is higher, I control my diet" was not endorsed by many participants. Therefore, we judged that content validity of this item was low and deleted it. A principal factor analysis and promax rotation were carried out for the remaining 26 items. The first factor "problem-solving" consisted of 10 items; the second factor "self-care behavior" consisted of 10 items; and the third factor "patient-health professions partnership" consisted of four items. Cronbach's alpha coefficients were sufficient: problem-solving $=0.87$, self-care behavior $=0.78$, and patient-health professions partnership $=0.74$. Table 1 shows the three factors identified by this factor analysis.

\section{The Multidimensional Scale of Perceived Social Support (MSPSS)}

The MSPSS was developed to measure conditions of social support. ${ }^{19}$ This study used the MSPSS Japanese version. ${ }^{20,21}$ The scale comprises 12 items and has three sources: family, friends, and a significant other. Cronbach's alpha coefficients for total, family, friends, and a significant other were 0.91 , $0.94,0.90$, and 0.88 , respectively. ${ }^{20}$ All subscales are evaluated on a 7-point Likert scale (1-7); high scores indicate better social support.

\section{The Maternal Consciousness Scale}

The Maternal Consciousness Scale measures acceptance of one's maternal role. ${ }^{17}$ Feelings are based on patients' answers to 12 questions that are categorized as "positive or active" and "negative or passive". Positive maternal role recognition (MP) is configured into six items: "I like the role of mother," "becoming a mother has provided me with growth," "you feel that you live the life you want when you behave as a mother," "I feel I have a purpose in life because I am a mother," "when I became a mother, I felt calm," and "I feel a pretty good sense of fulfillment as a mother." Negative maternal (MN) role recognition is determined by the following six items: "I feel child-rearing to be a burden," "I feel left behind from the world while I am involved with child-rearing," "my interests have narrowed because I am focused only on my children," "I do not think I am qualified as a mother," "I would be better off not having a child," and "I am limited in my activities because of being a mother." All subscales are evaluated on a 4-point Likert scale (1-4). High MP indicated a better situation, while high MN indicated an unfavorable situation. No prior reports regarding Cronbach's alpha coefficients were available for this scale, but the scale has been found to have good construct validity for MP and criterion-related validity for $\mathrm{MN} .{ }^{17}$

\section{Data analyses}

Mean and SD were calculated for all demographic data. A Mann-Whitney $U$ test was performed to compare employed and unemployed groups in terms of mean HRQOL scores. 
Table I Factor loadings for self-care behavior items

\begin{tabular}{|c|c|c|c|c|}
\hline \multicolumn{2}{|c|}{ Item } & Factor I & Factor 2 & Factor 3 \\
\hline \multicolumn{5}{|c|}{ Factor I: problem-solving (Cronbach's alpha=0.865) } \\
\hline Q4 & I pay attention to changes in creatinine values & 0.967 & -0.209 & -0.268 \\
\hline Q2 & When creatinine values are not ideal, I find out why & $0.88 \mathrm{I}$ & -0.280 & 0.168 \\
\hline Q7 & $\begin{array}{l}\text { When a decrease of urine or edema occurs, I spontaneously control my water absorption and } \\
\text { eat less salt }\end{array}$ & 0.667 & 0.097 & 0.013 \\
\hline Q5 & According to my symptoms, I will find the appropriate resources to handle them immediately & 0.660 & 0.007 & -0.047 \\
\hline QI & I continue to note blood-testing reports and compare them with suggestions given by my doctor & 0.623 & -0.106 & -0.178 \\
\hline Q3 & I gather information myself or ask other people questions & 0.593 & -0.083 & 0.060 \\
\hline Q22 & I immediately notice when I have unusual reactions or symptoms & 0.473 & 0.327 & 0.028 \\
\hline QII & I discuss with my doctor or nurse when I want to change my treatment plan & 0.436 & 0.133 & 0.260 \\
\hline Q14 & I prepare the questions I want answered beforehand and then consult my doctor & 0.425 & 0.333 & 0.052 \\
\hline Q12 & I consult my doctor if there is something I do not understand about my treatment & 0.379 & 0.039 & 0.217 \\
\hline \multicolumn{5}{|c|}{ Factor 2: self-care behavior (Cronbach's alpha $=0.78 \mathrm{I}$ ) } \\
\hline Q18 & I follow instructions given by my doctor or nurse for my transplant examination & 0.123 & 0.686 & -0.101 \\
\hline Q16 & I avoid going to public spaces where there are too many people, or the air quality is bad & -0.178 & $0.65 \mathrm{I}$ & -0.185 \\
\hline Q25 & When I do not feel comfortable, I think positively & -0.228 & 0.647 & 0.005 \\
\hline Q20 & I use measures to protect myself & 0.089 & 0.529 & -0.116 \\
\hline Q23 & I do not eat strong-flavored food & 0.074 & $0.5 \mathrm{I}$ & 0.018 \\
\hline Q24 & I measure my weight and blood pressure regularly & -0.152 & 0.486 & 0.140 \\
\hline Q26 & I take note of things that may make me feel uncomfortable during my daily life & 0.268 & 0.479 & 0.068 \\
\hline Q17 & I wear a mask when needed & -0.136 & 0.461 & 0.193 \\
\hline Q19 & I exercise regularly & -0.002 & 0.440 & -0.417 \\
\hline Q21 & $\begin{array}{l}\text { I always pay attention to my symptoms, like fever, body weakness, cough, sore throat, or pain in } \\
\text { my transplanted kidney }\end{array}$ & 0.329 & 0.357 & 0.180 \\
\hline \multicolumn{5}{|c|}{ Factor 3: patient-health professions partnership (Cronbach's alpha=0.74I) } \\
\hline Q10 & $\begin{array}{l}\text { If there are infection symptoms like fever, body weakness, cough, or sore throat, I follow } \\
\text { suggestions from my doctor or nurse }\end{array}$ & -0.144 & -0.185 & 0.899 \\
\hline Q13 & I feel comfortable when I talk to my doctor or nurse & -0.085 & 0.101 & 0.724 \\
\hline Q6 & $\begin{array}{l}\text { If there are rejection symptoms, like fever, decrease of urine, kidney pain, or high blood } \\
\text { pressure, I follow suggestions from my doctor or nurse }\end{array}$ & 0.145 & -0.034 & 0.568 \\
\hline Q8 & I contact my doctor or nurse, or go to the hospital, when I am not in a good condition & 0.038 & 0.214 & 0.410 \\
\hline \multicolumn{2}{|r|}{ Eigenvalue } & 7.28 & 2.37 & 1.99 \\
\hline \multicolumn{2}{|c|}{ Proportion of variance explained (\%) } & 30.32 & 9.86 & 8.27 \\
\hline \multicolumn{2}{|c|}{ Cumulative proportion of variance (\%) } & 30.32 & 40.18 & 48.45 \\
\hline \multicolumn{5}{|c|}{ Deleted items } \\
\hline Q9 & When blood sugar is higher, I control my diet & & & \\
\hline Q15 & I am willing to share my experiences with other kidney transplant patients & & & \\
\hline Q27 & When I am in trouble, I seek help & & & \\
\hline
\end{tabular}

Note: Values in bold indicate the highest loading.

Spearman's rank correlation coefficients were employed to assess the relationships between the variables and HRQOL, because Spearman's correlations used ordinal measurement like in SF-36 and in each scale. The initial model was based on two hypotheses: social support and maternal recognition are positively and significantly related to self-management and self-management is positively significantly related to QOL. Structural equation modeling (SEM) was adopted to assess the fit of an initial model to the data. If the initial model fit was poor, model improvement was accomplished by adding or deleting certain paths. Adequate model fit was determined by an adjusted goodness-of-fit index of $>0.80$ and a root mean square error of approximation $<0.10$.
SPSS Statistics 22 (IBM Corporation) and SPSS Amos 23 (IBM Corporation) were used for the analyses.

\section{Results}

We obtained cooperation from 21 of the 200 kidney transplant centers that we contacted. Given that there are fewer kidney transplant centers in Japan relative to the USA and Europe, many of the Japanese centers had no patients who had given childbirth after a transplant. Overall, we were able to recruit 83 participants. Of the 83 questionnaires that were distributed, 65 (78\%) were returned. Six respondents had missing data; thus, data from 59 (71\%) respondents were available for the analyses. 


\section{Participant characteristics}

Recipient characteristics are shown in Table 2. Most participants experienced immunoglobulin A nephropathy prior to transplantation (54\%). In all, 95\% of the kidneys transplanted were from living donors, $3.4 \%$ from deceased donors, and $1.7 \%$ from unknown donors. The average number of family members of transplant recipients was $3.7 \pm 1.1$, and the number of children was $1.36 \pm 0.6$. Eighty-two fetal outcomes were evaluated. Maternal age was $33.6 \pm 4.1$ years, duration of gestational period was $35.3 \pm 3.3$ weeks, and birth weight was $2,303.8 \pm 592.5 \mathrm{~g}$.

\section{Participant psychometric characteristics}

Table 3 shows the HRQOL, self-management, MSPSS, and Maternal Consciousness scale scores. The mean PCS score was $49.6 \pm 10.2$, the MCS score was $48.4 \pm 7.8$, and the RCS score was $50.6 \pm 11.4$.

\section{Comparisons and relationships between scales}

Factors affecting HRQOL following kidney transplantation included employment. Thus, it was necessary to examine the difference between an employed and an unemployed group. An employed and an unemployed group were compared using a Mann-Whitney $U$ test for each HRQOL subscale. This test showed no significant differences between the groups on any score: PCS $(p=0.48), \operatorname{MCS}(p=0.94)$, and $\operatorname{RCS}(p=0.33)$ scores.

Each HRQOL subscale, self-management, MSPSS, and Maternal Consciousness scale scores were analyzed using

Table 2 Demographics and baseline characteristics of the participants $(\mathrm{N}=59)$

\begin{tabular}{ll}
\hline Variables & Mean \pm SD \\
\hline Age, years & $42.3 \pm 7.2$ \\
Age at transplant, years & $28.2 \pm 4.6$ \\
Age at first childbirth after transplant, years & $33.6 \pm 4.1$ \\
Serum creatinine level, mg/dL & $\mathrm{I} .4 \pm \mathrm{I} .0$ \\
Education, $\mathrm{n}(\%)$ & \\
$\quad$ College and university or higher & $39(66 . \mathrm{I})$ \\
High school & $\mathrm{I}(28.8)$ \\
Junior high school or less & $2(3.4)$ \\
Unknown & $\mathrm{I}(\mathrm{I} .7)$ \\
Employment status, $\mathrm{n}$ (\%) & \\
$\quad$ Employed & $3 \mathrm{I}(52.5)$ \\
Unemployed & $27(45.8)$ \\
$\quad$ Unknown & $\mathrm{I}(\mathrm{I} .7)$ \\
Donor type, $\mathrm{n}(\%)$ & \\
Living & $56(94.9)$ \\
Deceased & $2(3.4)$ \\
Unknown & $\mathrm{I}(\mathrm{I} .7)$ \\
\hline
\end{tabular}

Table 3 Descriptive statistics for the study variables $(\mathrm{N}=59)$

\begin{tabular}{ll}
\hline Variables & Mean \pm SD \\
\hline Health-related quality of life & \\
Physical functioning & $51.2 \pm 7.9$ \\
Role physical & $48.5 \pm 9.4$ \\
Bodily pain & $53.4 \pm 9.2$ \\
Social functioning & $50.7 \pm 8.9$ \\
General health perceptions & $46.1 \pm 9.6$ \\
Vitality & $47.3 \pm 8.9$ \\
Role emotional & $50.6 \pm 8.9$ \\
Mental health & $51.6 \pm 7.8$ \\
Physical component summary & $49.6 \pm 10.2$ \\
Mental component score & $48.4 \pm 7.8$ \\
Role/social component score & $50.6 \pm I I .4$ \\
Self-management & \\
Problem-solving (range=0-40) & $27.9 \pm 8.2$ \\
Self-care behavior (range=0-40) & $25.5 \pm 5.6$ \\
Patient-health professions partnership (range $=0-16)$ & $13.4 \pm 2.6$ \\
Social support (MSPSS) & $6.0 \pm 0.8$ \\
Family & $6.1 \pm 0.8$ \\
Friends & $5.5 \pm I .4$ \\
Significant other & $6.3 \pm 0.7$ \\
Maternal consciousness & \\
Positive consciousness & $3.0 \pm I .4$ \\
Negative consciousness & $1.4 \pm 0.4$ \\
\hline Ab & \\
\hline
\end{tabular}

Abbreviation: MSPSS, Multidimensional Scale of Perceived Social Support.

Spearman's rank correlations. Results showed that PCS was positively correlated with the MSPSS ( $r=0.29, p=0.025$ ); self-care behavior was positively correlated with the MCS $(r=0.29, p=0.029)$, MSPSS $(r=0.31, p=0.016)$, MP $(r=0.44$, $p=0.001)$, and problem-solving scale $(r=0.43, p=0.001)$. Furthermore, serum creatinine level was negatively correlated with the PCS $(r=-0.29, p=0.029)$ and MCS $(r=-0.42$, $p=0.001)$. There were no statistically significant effects of time since kidney transplant and time since first childbirth after transplant on any HRQOL scale or self-management scores. Correlation results are shown in Table 4.

\section{Model goodness-of-fit}

The final SEM model is shown in Figure 1. The fit indices are shown below. The adjusted goodness-of-fit index was 0.82 , the goodness-of-fit index was 0.92 , and the comparative fit index was 0.89 . The root mean square error of approximation was 0.08 ; overall, these values are generally acceptable. Here, PCS was influenced by MSPSS, and MSPSS was influenced by self-care behavior and patient-health professions partnership. MCS was influenced by self-care behavior, and MP was influenced by problem-solving and self-care behavior. The coefficient for the correlation between problem-solving and self-care behavior was 0.40 . The coefficient for the 


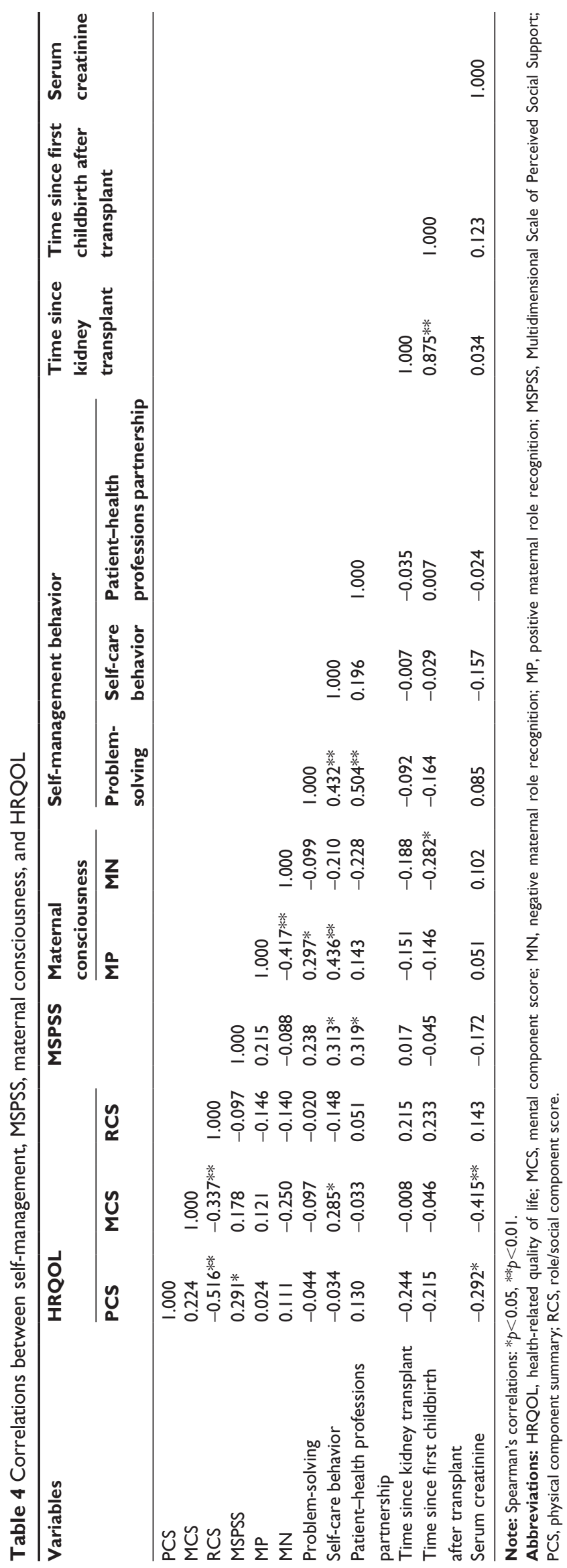

correlation between problem-solving and patient-health professions partnership was 0.33 . Self-management did not differ significantly based on PCS. However, self-care behavior and patient-health professions partnership appeared to indirectly affect physical health through social support.

\section{Discussion}

The present study investigated HRQOL post-childbirth among kidney transplant recipients. Additionally, we elucidated factors associated with HRQOL among this sample. To our knowledge, this is the first study to collect QOL data on $>50$ patients, who had given birth post-kidney transplantation. Hence, we obtained several novel findings. One surprising result was that self-care behaviors and patienthealth professions partnership indirectly affected physical health through social support.

Overall HRQOL was satisfactory for the present sample, as HRQOL tends to be comparable among kidney transplant recipients and the general population. ${ }^{14}$ However, role physical, general health, and vitality mean score values were lower in the present sample. It is important to assess physical HRQOL, as this can be an influential contributor to adaptive functioning within this patient population. Serum creatinine distribution is a significant contributor to kidney graft success. Consistent with previous research, ${ }^{6,22}$ only serum creatinine level was related to both physical and mental HRQOL. However, serum creatinine level was not associated with the main factors of interest in the present study, including self-management, and self-management was not related to physical or mental HRQOL. This was an interesting result, given the fact that self-management is very important for kidney transplant recipients, and as medical professionals believe that self-management allows for effective maintenance of serum creatinine levels. Owing to the highrisk nature of pregnancy in posttransplant patients, it may be of benefit to review the importance of self-management among transplant recipients to ensure maintenance of renal function. Since transplant recipients experience significant anxiety regarding their pregnancy and delivery, ${ }^{23-25}$ methods for improving confidence with self-care management should be addressed in future research.

The present study also revealed that self-care behavior and patient-health professions partnership indirectly affected physical health through social support. Additionally, problem-solving affected both self-care behavior and the patient-provider health professions partnership. Previous studies report that HRQOL tends to be higher among recipients living near others (eg, married couples or cohabitants) 


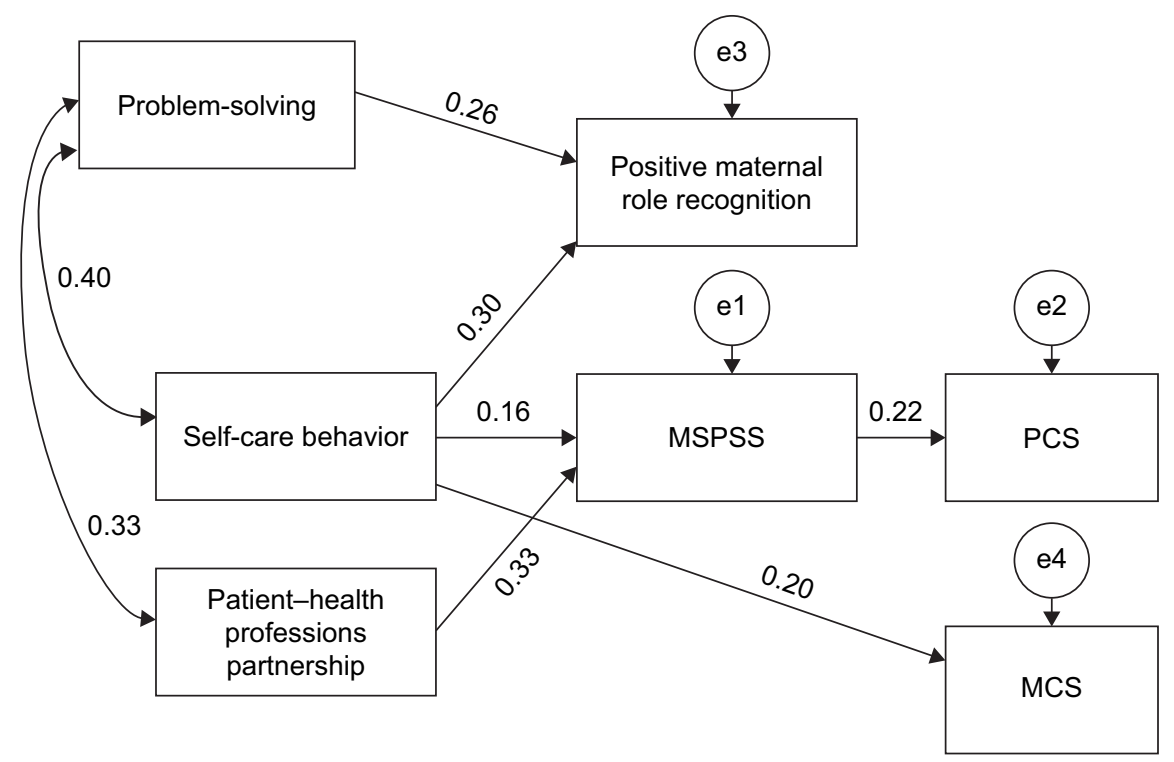

Figure I The final model of HRQOL of women who gave birth after kidney transplantation.

Notes: The GFI was 0.92 , the AGFI was 0.82 , and the CFI was 0.89 . The RMSEA was 0.08 . el-e4 shows the coefficients for errors.

Abbreviations: AGFI, adjusted goodness-of-fit index; CFI, comparative fit index; GFI, goodness-of-fit index; HRQOL, health-related quality of life; MCS, mental component score; MSPSS, Multidimensional Scale of Perceived Social Support; PCS, physical component summary; RMSEA, root mean square error of approximation.

than among recipients living alone. ${ }^{3}$ It is important for recipients to have reliable family members to aid with care and support. Furthermore, if a kidney recipient wishes to become pregnant, medical staff must confirm prerequisites for self-management, particularly in terms of available family support. In addition to the support available, recipients may benefit from increasing their problem-solving abilities, which appear to have led to improved self-care behavior and patient-health professions partnership associations in the present study. Thus, the combined effects of recipients working to improve their problem-solving skills, along with reasonable reliance on a social support network, could have immense benefits for transplant recipients looking to become mothers.

Observed maternal role recognition was rather positive/ active in the present study. Furthermore, positive role recognition was positively correlated with self-care behavior and patient-health professions partnership. Previous research observed that parental stress is strongly related to $\mathrm{MN}$, and maternal stress is greatly affected by satisfaction with paternal childcare involvement. ${ }^{18}$ Furthermore, stress is exacerbated when experiencing the birth and care of a first child. ${ }^{18}$ In Japan, there is a cultural tendency for fathers to be less involved in parenting, while the mother is expected to be wholly responsible for parenting. Thus, to alleviate the compounding stressors experienced by mothers with a renal transplant, fathers need to be more involved with child-rearing duties.
Additionally, in this study, the fact that HRQOL was not related to employment among kidney transplant recipients is contrary to what has been observed in previous studies. ${ }^{4,5}$ One key reason for this difference is the fact that we limited our participant pool to mothers who had given birth posttransplantation. This finding seems plausible, given the importance of the role of social support in Japanese mothers and on maternal care and child-rearing. Regardless of the employment status of the mother, it can be interpreted that the patient's role as a mother is independent of her QOL. A role as a mother may transcend the employment status. Although pregnancy leads to increased risks for the transplant recipient, having a partner and being a parent may lead to improvements in QOL.

We initially hypothesized that the driving factors in determining QOL in mothers who had a posttransplant pregnancy were social support and maternal consciousness, which, in turn, influenced self-management, thus improving QOL. It was predicted that a mother's instinct to want to maintain good health to care for her child was what led to self-management behaviors. However, our results contradict the original hypothesis. Contrary to our initial hypotheses, the final SEM model in this study showed that this foundational self-management behavior promoted maternal consciousness and social support, which subsequently influenced physical QOL. This suggests that self-management behaviors in renal transplant recipients preceded pregnancy and childbirth and served as a foundation for parenthood. Education in 
the posttransplant period is essential to promote patients' health maintenance. Based on the results of this study, improvement in problem-solving skills leads to an increase in self-care behavior and encourages a synergistic patientdoctor relationship. Problem-solving skills have also been found to promote help-seeking behavior when the recipient is faced with difficult situations. Moreover, with help from others, the recipient can reaffirm her maternal role, thereby encouraging maintenance of self-care and ultimately improving HRQOL. For the recipient, living with children poses myriad challenges including exposure to childhood infections, inconsistent medication use, and inadequate sanitary environment. For the recipient to overcome these challenges of parenthood, it is critical to improve problem-solving skills and create an environment where the recipient can reach out to others for assistance.

A few limitations of the study should be noted. First, given the cross-sectional nature of the present study, transitional/ maturational aspects of HRQOL could not be determined in this transplant sample. Second, prior studies have observed general sex differences in the quality of physical life after a kidney transplant, with women reporting diminished quality. Since all participants were women, we cannot be certain that the observed level of HRQOL was due to child-rearing experiences/duties or because of being a woman. Therefore, comparisons of pre- and posttransplantation would be necessary to assess whether changes to HRQOL are unique to women who do or do not give birth after their transplant. Third, owing to the small size of the patient cohort in this study, bias control is inadequate. As posttransplantation pregnancy cases would increase in the future, it becomes necessary to conduct interventional research with an appropriate control group. Finally, future work should assess how child-rearing duties impact men who become fathers after a transplant to obtain a more comprehensive assessment of how parenting, in general, impacts HRQOL.

Pregnancy after kidney transplantation is associated with increased risks for maternal and fetal complications. ${ }^{7-11}$ However, the present study revealed that women experiencing a pregnancy and child-rearing after a kidney transplant had comparable levels of HRQOL relative to the general population. This may be partly because of the role of selfmanagement that indirectly influenced physical health through social support. Therefore, supportive environments created through monitoring patients and consulting transplant professionals and recipient families may increase self-management, which, in turn, might improve HRQOL. Thus, when a kidney transplant patient is considering pregnancy and childbirth, it is important for her to have well-developed self-management skills and a supportive environment in place.

\section{Conclusion}

The present study elucidated factors associated with HRQOL among kidney transplant recipients who have given birth posttransplantation. Serum creatinine level was related to both physical and mental HRQOL. Furthermore, selfmanagement behavior affected social support and maternal role recognition. Finally, the study revealed that women who experienced pregnancy and child-rearing after transplantation reported sufficient HRQOL, particularly through indirect associations between self-management, physical health, and social support. Social support for self-management may contribute to improve HRQOL for women who experience pregnancy and child-rearing after transplantation.

\section{Acknowledgments}

We thank the participants for their cooperation, and we wish to express our appreciation to the transplant center professionals for their assistance. We would also like to thank Editage (www.editage.com) for their English language editing and publication support. This study was supported by the Japan Society for the Promotion of Science, Grants-in-Aid for Scientific Research Number JP25870952.

\section{Disclosure}

The authors report no conflicts of interest in this work.

\section{References}

1. Weng LC, Dai YT, Huang HL, Chiang YJ. Self-efficacy, self-care behaviours and quality of life of kidney transplant recipients. J Adv Nurs. 2010;66(4):828.

2. Liu H, Feurer ID, Dwyer K, Speroff T, Shaffer D, Wright Pinson C. The effects of gender and age on health-related quality of life following kidney transplantation. J Clin Nurs. 2008;17(1):82.

3. Neipp M, Karavul B, Jackobs S, et al. Quality of life in adult transplant recipients more than 15 years after kidney transplantation. Transplantation. 2006;81(12):1640.

4. Wei TY, Chiang YJ, Hsieh CY, Weng LC, Lin SC, Lin MH. Health related quality of life of long-term kidney transplantation recipients. Biomed J. 2013;36(5):243.

5. Chen WC, Chen CH, Lee PC, Wang WL. Quality of life, symptom distress, and social support among renal transplant recipients in Southern Taiwan: a correlational study. J Nurs Res. 2007;15(4):319.

6. Bohlke M, Marini SS, Rocha M, et al. Factors associated with health-related quality of life after successful kidney transplantation: a population-based study. Qual Life Res. 2009;18(9):1185.

7. Coscia LA, Constantinescu S, Moritz MJ, et al. Report from the National Transplantation Pregnancy Registry (NTPR): outcomes of pregnancy after transplantation. Clin Transpl. 2010;65-85.

8. Fuchs KM, Wu D, Ebcioglu Z. Pregnancy in renal transplant recipients. Semin Perinatol. 2007;31(6):339.

9. Gill JS, Zalunardo N, Rose C, Tonelli M. The pregnancy rate and live birth rate in kidney transplant recipients. Am J Transplant. 2009; 9(7):1541. 
10. Deshpande NA, James NT, Kucirka LM, et al. Pregnancy outcomes in kidney transplant recipients: a systematic review and meta-analysis. Am J Transplant. 2011;11(11):2388.

11. Blume C, Sensoy A, Gross MM, et al. A comparison of the outcome of pregnancies after liver and kidney transplantation. Transplantation. 2013;95(1):222.

12. The Japan Society for Transplantation, Japanese Society for Clinical Renal Transplantation. Annual progress report from the Japanese Renal Transplant Registry: number of renal transplantation in 2015 and follow-up survey. JPN J Transplant. 2016;51(2-3):124.

13. Mitani M, Matsuda Y. Reproductive endocrinology and pregnancy in renal transplant recipients. Sanfujinka Chiryo. 2011;102:717.

14. Fukuhara S, Suzukamo Y. Manual of SF-36v2 Japanese Version. Kyoto: Institute for Health Outcomes \& Process Evaluation Research; 2004.

15. Hayashi Y. The relationships between the components in QOL causal model of kidney posttransplant recipients and recipient characteristics. Bull Sch Health Sci Okayama Univ. 1997;8(1):61.

16. Kosaka S, Tanaka M, Sakai T, et al. Survey of self-management in kidney transplant recipients and its association with their post-transplantation period. Jpn J Transplant. 2012;47(1):60.

17. Ohinata M. Study of motherhood: The formation and the process of the change: Counterevidence to a traditional motherhood view. Kawashima Shoten. Tokyo: 1988. Available from: http://iss.ndl.go.jp/ books/R100000002-I000001937296-00?ar=4e1f\&locale=en. Accessed May 10, 2018. Japanese.
18. Kuwana K, Hosokawa $\mathrm{T}$. The parenting stress felt by parents of 18-month-old children (1) -contributing factors to mother's parenting stress. Annu Bull Graduate Sch Educ Tohoku Univ. 2007;56(1):247.

19. Zimet GD, Dahlem NW, Zimet SG, Farley GK. The multidimensional scale of perceived social support. J Pers Assess. 1988;52(1):30.

20. Iwasa H, Gondo Y, Masui Y, et al. Reliability and validity of Japanese version "Social support scale"-study for middle-aged and elderly people. J Health Welf Stat. 2007;54(6):26.

21. Endo S, Oish K. The influence of social support of friends and ikigai on depressive tendencies in college students. Jpn J Pers. 2015; 24(2):102.

22. Griva K, Davenport A, Newman SP. Health-related quality of life and long-term survival and graft failure in kidney transplantation: a 12-year follow-up study. Transplantation. 2013;95(5):740.

23. Yoshimura Y, Umeshita K, Kubo S, Yoshikawa Y. Anxieties and coping methods of liver transplant recipients regarding pregnancy and delivery. $J$ Adv Nurs. 2016;72(8):1875.

24. Tong A, Brown MA, Winkelmayer WC, Craig JC, Jesudason S. Perspectives on pregnancy in women with CKD: a semistructured interview study. Am J Kidney Dis. 2015;66(6):951.

25. Tong A, Jesudason S, Craig JC, Winkelmayer WC. Perspectives on pregnancy in women with chronic kidney disease: systematic review of qualitative studies. Nephrol Dial Transplant. 2015;30(4):652.
International Journal of Women's Health

\section{Publish your work in this journal}

The International Journal of Women's Health is an international, peerreviewed open-access journal publishing original research, reports, editorials, reviews and commentaries on all aspects of women's healthcare including gynecology, obstetrics, and breast cancer. The manuscript management system is completely online and includes

\section{Dovepress}

a very quick and fair peer-review system, which is all easy to use. Visit http://www.dovepress.com/testimonials.php to read real quotes from published authors. 